\section{Zoonosis bacterianas de aparición reciente ${ }^{1}$}

Palabras clave: zoonosis, enfermedades emergentes, enfermedades reemergentes.

\footnotetext{
Basado en la siguiente fuente: Chomel BB. Emerging bacterial zoonoses. Ponencia presentada en la Universidad de Nihon, Japón, el 29 de agosto de 2000 y en la Universidad de Rakuno Gakuen, Hokkaido, Japón, el 1 de septiembre de 2000. Bruno B. Chomel está afiliado con el Centro Colaborador de la OMS en Zoonosis Nuevas y Emergentes, Departamento de Salud Poblacional y Reproducción, Escuela de Medicina Veterinaria, Universidad de California en Davis, California. Dirección postal: School of Veterinary Medicine, University of California, Davis, CA 95616, EE.UU.
}

El siglo $X X$ vio el recrudecimiento de algunas infecciones bacterianas que se daban por controladas, como la tuberculosis, y de zoonosis bacterianas de muy diversa índole: 1) las de origen alimentario, como las producidas por Salmonella, bacterias coliformes o Campylobacter; 2) las que se transmiten por contacto con animales domésticos o salvajes, como la peste, la fiebre $\mathrm{Q}$ (producida comúnmente por Coxiella [o Rickettsia] burnetii) y algunas salmonelosis; 3) las que se asocian con estados inmunodepresivos, como la neumonía por Rhodococcus equi o la angiomatosis bacilar causada por Bartonella henselae, y 4) las zoonosis transmitidas por vectores, tales como la enfermedad de Lyme, las erliquiosis humanas o la enfermedad de la fiebre botonosa del Mediterráneo.

La mayor parte de las enfermedades que ahora se observan en humanos por primera vez - como las provocadas por el virus Ebola o del Nilo Occidental — también afectan a los animales. De estas enfermedades emergentes hay dos tipos: las de identificación reciente, como las infecciones por Capnocytophaga canimorsus o Escherichia coli O157:H7, y las que habían sido identificadas clínicamente con anterioridad sin que se hubiese identificado su agente causal. Tal es el caso de la enfermedad del arañazo del gato y de las erliquiosis en seres humanos.

Por otra parte, una zoonosis se considera reemergente cuando aumenta bruscamente el número de casos en seres humanos o animales, lo cual ocurre habitualmente por alteración de algún factor ambiental. Las brucelosis causadas por Brucella melitensis, la tularemia y la listeriosis pertenecen a este grupo.

\section{IDENTIFICACIÓN DE LOS AGENTES CAUSALES}

La actual capacidad para identificar los agentes causales de las enfermedades emergentes y reemergentes obedece, en gran medida, a las extraordinarias herramientas que ha puesto a nuestro alcance la biología molecular, particularmente la técnica de la reacción en cadena de la polimerasa (RCP) y la secuenciación génica. Entre las nuevas zoonosis que han sido identificadas mediante estas técnicas figuran las que son provocadas por los hantavirus del Nuevo Mundo y la angiomatosis bacilar causada 
por Bartonella henselae. Gracias a estas técnicas, es ahora más factible cuantificar la carga zoonótica en el reino animal y en comunidades humanas.

\section{VÍAS DE TRANSMISIÓN}

Las fuentes de transmisión más frecuentes de las zonosis emergentes y reemergentes están constituidas por los alimentos (salmonelosis, colibacilosis [E. coli O157:H7]; campilobacteriosis, listeriosis y brucelosis (Brucella melitensis)] y el aire (fiebre Q [Coxiella burnetti], Streptococcus suis y Rhodococcus equi) contaminados, la picadura del insecto vector (enfermedad de Lyme, erliquiosis, Rickettsia felis y Bartonella henselae), y el arañazo o mordedura de un animal infectado (enfermedad del arañazo del gato, Capnocytophaga canimorsus, Mycobacterium marinum y Streptococcus iniae).

\section{FACTORES QUE PROPICIAN LA APARICIÓN Y REAPARICIÓN DE ALGUNAS ENFERMEDADES}

Tan numerosas son las enfermedades que han surgido y resurgido en los últimos decenios, que nombrarlas representa un ejercicio estéril. Más provechoso desde el punto de vista de su prevención y control es entender qué factores favorecen su diseminación.

Se cree que el surgimiento de zoonosis nuevas, o el recrudecimiento de enfermedades ya conocidas, obedece a factores tan diversos como cambios climáticos y ambientales; el carácter internacional de la producción y distribución de alimentos; factores demográficos; el desplazamiento de seres humanos de un lugar a otro; la adaptación de los agentes causales a nuevas condiciones ecológicas, y la deficiencia de las medidas de control.

Como puede verse, los factores que propician la transmisión varían según el agente causal. Cabe tener presente que la población del mundo ha sufrido un aumento vertiginoso a lo largo del siglo XX, sobre todo en los países en desarrollo, y este fenómeno se ha acompañado de la desaparición de grandes zonas forestales, del cultivo de zonas agrícolas previamente baldías, de un aumento de los viajes, sobre todo de tipo turístico, y de la formación de vastas metrópolis donde el hacinamiento y las condiciones de vida precarias dan origen a focos de transmisión. Otro factor que contribuye a la aparición de nuevas infecciones, especialmente en países desarrollados, es el envejecimiento de la población, puesto que las personas de edad avanzada son, por lo general, más susceptibles a las enfermedades (1-3).
Muchas enfermedades nuevas y reemergentes tienen como reservorio un animal salvaje que transmite la infección a especies domésticas y al ser humano, bien sea por contacto directo o por conducto de un vector (tal es el caso de las erliquiosis y de la enfermedad de Lyme). Ciertos hábitos que se ven facilitados por los viajes y la erosión de fronteras culturales, como la ingestión de comidas internacionales y la tenencia doméstica de animales exóticos, generan una mayor exposición del hombre a los agentes infecciosos que producen enfermedades emergentes o reemergentes (1-3).

\section{Cambios de carácter ambiental}

Cualquier cambio ambiental - la deforestación o reforestación, la irrigación de cultivos a gran escala, la urbanización, etc.- - altera la distribución de reservorios y vectores y de las especies de animales infectadas.

Lógicamente, la llegada a un territorio de un vector, reservorio o agente infeccioso que previamente no habitaba en él acarrea el riesgo de que surjan o resurjan algunas zoonosis. Esto ocurrió, por poner un ejemplo, cuando al final del siglo XIX hubo una importación a Europa Occidental de liebres infectadas procedentes de Europa Central, con la consiguiente aparición de casos de tularemia (4). Los animales salvajes constituyen una gran masa susceptible de ser infectada y de dar origen a nuevas afecciones. De 1973 a 1986, se produjeron en Estados Unidos, Canadá, Australia y Nueva Zelandia alrededor de 700 traslados de animales de un territorio a otro, y en $24 \%$ de los casos estos se efectuaron sin tomar medidas para la detección de agentes infecciosos (5).

\section{Elaboración de alimentos y comercio a escala internacional}

Las enfermedades que se transmiten por los alimentos afectan a millones de personas debido, entre otras cosas, a la creciente popularidad de alimentos "de preparación rápida" y la vasta distribución comercial de productos alimentarios.

\section{Estados de inmunodepresión}

La inmunosupresión debida a la infección por VIH o a la quimioterapia y al tratamiento con esteroides se suma al envejecimiento como factor que predispone a la infección por ciertos microorganismos. El contacto con gatos portadores de Bartonella 
henselae puede provocar la aparición de angiomatosis bacilar en pacientes con sida avanzado. El envejecimiento de la población en países desarrollados, como se señalaba anteriormente, contribuye a la aparición de zoonosis nuevas que suelen ser asintomáticas o menos graves en personas jóvenes. Las vías de transmisión y los agentes causales son sumamente variados.

Transmisión respiratoria. Entre las zoonosis que se transmiten por la vía respiratoria, dos se observan comúnmente en personas infectadas por el VIH. Estas son las causadas por Rhodococcus equi, cuya tasa de letalidad oscila entre 25 y $50 \%$, y por Bordetella bronchiseptica, bacilo Gram-negativo que forma parte de la flora bucal del perro y que produce la llamada "tos canina" $(6,7-9)$. Las personas inmunodeprimidas no deben asistir a competencias caninas y deben vacunar a sus perros domésticos para reducir el riesgo de transmisión (10).

Transmisión por mordedura, arañazo o trauma. Capnocytophaga canimorsus es una bacteria Gram-negativa que se encuentra en la cavidad bucal de $16 \%$ de los perros sanos. La infección en el ser humano, que produce una pneumonía cavernosa con una tasa de letalidad de $30 \%$, suele ser causada por mordedura $(11,12)$. El primer caso se notificó en 1976 y desde entonces se han notificado más de 100 en Europa, América del Norte y Australia (11). En personas que no están inmudeprimidas, la infección es asintomática, pero produce manifestaciones en personas mayores de 50 años, personas que han sido sometidas a esplenectomía, alcohólicos, o personas que reciben un tratamiento inmunosupresor. Esta infección debe sospecharse en cualquier persona inmunodeprimida con un cuadro febril después de ser mordida por un animal, particularmente un perro (13). La septicemia se produce en $94 \%$ de estos casos. El agente causal es susceptible a casi todos los antibióticos y el más indicado es la penicilina procaínica (11).

Angiomatosis bacilar por Bartonella henselae. El descubrimiento entre 1983 y 1988 de casos de angiomatosis bacilar, caracterizada por lesiones proliferativas, principalmente en personas con sida avanzado, llevó al aislamiento de una nueva bacteria, Bartonella (previamente conocida por Rochalimaea) henselae. Por accidente, esta bacteria también se identificó como el agente causal de la fiebre por arañazo del gato. La enfermedad cursa con lesiones cutáneas nodulares o papulares que se asemejan a las del sarcoma de Kaposi. También puede haber lesio- nes viscerales $\mathrm{u}$ óseas. Cuando la enfermedad está diseminada en el organismo, los síntomas principales son fiebre, malestar general, pérdida de peso e hipertrofia de los órganos afectados. También se han documentado casos de endocarditis (14).

Infección por Mycobacterium marinum. Mycobacterium marinum ha sido identificado como agente causal de una infección granulomatosa que suele afectar a pacientes de sida que tienen peces decorativos en su casa. La transmisión en estas personas se produce cuando limpian el acuario sin ponerse guantes y suele ser sistémica. En personas que no están inmunodeprimidas, la lesión granulomatosa se limita al sitio de inoculación (6).

\section{Zoonosis transmitidas por vectores}

En años recientes, diversas zoonosis bacterianas transmitidas por vectores han sido identificadas en distintas partes del mundo como consecuencia de cambios ambientales provocados por la reforestación y creación de nuevos hábitat periurbanos, y por el desarrollo de mejores técnicas diagnósticas. Entre ellas figuran la enfermedad de Lyme, las erliquiosis que afectan a seres humanos y algunas zoonosis reemergentes, tales como la fiebre botonosa del Mediterráneo.

Enfermedad de Lyme. Esta enfermedad, producida por la espiroqueta Borrelia burgdorferi, se transmite por la picadura de garrapatas del género Ixodes: en Europa por I. ricinus y en Estados Unidos por I. scapularis e I. pacificus (en el nororiente y oriente central del país, y en la parte occidental, respectivamente). De las enfermedades transmitidas por vectores, esta representa 95\% de las que se notifican en Estados Unidos, donde anualmente ocurren alrededor de 12500 casos en humanos. Desde que se inició la vigilancia de la enfermedad en 1982, el número anual de casos ha aumentado casi 25 veces, debido en toda probabilidad a la marcada proliferación del vector en zonas con un gran aumento de la población de roedores y venados de donde aquel deriva su alimento. El progresivo abandono de la agricultura tradicional en Nueva Inglaterra y la reforestación de las tierras que han quedado en desuso han generado una proliferación desmesurada del venado en ese territorio (15).

Las erliquiosis. Estas zoonosis transmitidas por garrapatas han sido identificadas en años recien- 
tes. En 1986, se describió en Estados Unidos por primera vez una erliquiosis monocítica que se atribuyó inicialmente a Ehrlichia canis pero que más tarde se asoció con E. chaffeensis. Desde entonces, más de 700 casos han sido notificados en ese país, principalmente en los estados sureños centrales y orientales (16).

Más recientemente se ha documentado la presencia de una erliquiosis humana producida por $E$. canis, y el agente de la erliquiosis canina granulomatosa, E. ewingii, también ha sido identificado hace relativamente poco en cuatro pacientes (17, 18). Otras erliquiosis de notificación reciente son las causadas por organismos similares a E. phagocytophilia y E. equi y por I. scapularis (19).

Cada tipo de erliquiosis monocítica o granulomatosa se acompaña de uno o más síntomas que permiten identificarla: fiebre, escalofríos, cefalea, mialgia, anorexia y erupción cutánea, leucopenia, trombocitopenia, anemia, hipotensión, insuficiencia renal, coagulopatías, pancitopenia y elevación de las transferasas hepáticas $(16,20)$. La tasa de letalidad puede ascender a $5 \%$ en el caso de la erliquiosis monocítica y a $10 \%$ en el de la erliquiosis granulocítica (16). El diagnóstico se establece primariamente por inmunofluorescencia en suero o por la RCP, y el tratamiento consiste en la administración de doxicilina (100 mg dos veces al día durante 10 a 14 días). Como medidas preventivas se recomiendan el uso de ropa protectora e insecticidas y el examen cuidadoso de la piel en las partes del cuerpo que están descubiertas.

Fiebre botonosa del Mediterráneo. La fiebre botonosa del Mediterráneo (FBM) es una infección aguda cuyo agente causal es Rickettsia conori (21). Su reservorio principal es la garrapata canina de color pardo conocida por Rhipicephalus sanguineus, que raras veces se alimenta del ser humano durante su vida adulta pero que lo hace en ocasiones durante las fases más tempranas de su desarrollo. Se ha detectado un aumento de casos en seres humanos en los últimos 10 a 15 años, especialmente en el sur de Francia y en España, y el perro parece desempeñar un papel importante en la epidemiología de la enfermedad en seres humanos (22).

La infección humana se manifiesta por una lesión oscura característica en el sitio donde se adhiere la garrapata, habitualmente con tumefacción de un ganglio linfático. Alrededor de una semana más tarde, se producen fiebre y cefalea intensa, mialgias y dolores articulares, y pocos días después aparece una erupción cutánea de tipo macular o maculopapular que dura aproximadamente una semana (22). Varios estudios han establecido una asociación entre la incidencia de casos en seres humanos, por un lado, y el grado de infestación por garrapatas o la población canina por el otro $(21,22)$. También hay una posibilidad de que las condiciones socioeconómicas de una población determinada ejerzan un efecto desfavorable en la higiene personal y que por ese medio aumenten el riesgo de infección.

Síndrome tifoideo causado por Rickettsia felis. Rickettsia felis fue descubierto por microscopia electrónica en 1990 en la pulga felina, Ctenocephalides felis. De cada cinco casos de tifus murino diagnosticados en el estado de Texas, Estados Unidos, uno fue atribuido a este microorganismo sobre la base de los resultados de la prueba de RCP. La enfermedad producida por esta bacteria se parece al tifus murino, cuyo agente causal más común, Rickettsia typhi, es endémico en el suroeste de Estados Unidos, donde su principal reservorio salvaje es la comadreja. Este animal suele ser susceptible a la infestación por la garrapata felina en casi todo el país. Una encuesta serológica de gatos con fiebre en el noreste de Estados Unidos reveló la presencia de anticuerpos contra $R$. felis en $8 \%$ de estos gatos, lo cual indica que el gato doméstico podría desempeñar algún papel en la transmisión de la infección a seres humanos (23).

\section{El contacto con animales domésticos}

El caso de la peste sirve para ilustrar el papel que pueden desempeñar los animales domésticos en la aparición o reaparición de enfermedades. Antes de 1977, ningún caso de peste en Estados Unidos se había asociado con la exposición a gatos domésticos, pero desde entonces se han notificado 18 casos atribuibles al contacto con gatos infectados. De estos casos, más de la cuarta parte se presentaron en veterinarios o sus empleados. Más de $25 \%$ de estos casos en humanos fueron de tipo pulmonar. Desde que la peste apareció en California a principios del siglo $X X$, su distribución se ha venido desplazando hacia el este del país. Se cree que el aumento de la población en zonas periurbanas, con el consiguiente incremento de la población felina, explica este fenómeno y el aumento de casos de peste en gatos (24).

Y los animales exóticos, como ciertos peces de acuario, las iguanas y algunos reptiles, han dado origen a más de un caso grave y hasta mortal de salmonelosis en niños pequeños. En el Reino Unido, un neonato murió en 1999 de meningitis por Salmonella rubislaw; en los últimos dos años, 13 casos de salmonelosis en seres humanos han sido diagnosticados en conexión con la tenencia de reptiles en el hogar. En Canadá, alrededor de 3 a 5\% de todos los 
casos de salmonelosis se asocian con la exposición a reptiles o anfibios (25). Más de $90 \%$ de los reptiles son portadores de tipos raros o desconocidos de Salmonella y excretan la bacteria. Las iguanas se infectan por ingesta de materia fecal de seres humanos, proceso natural que les permite desarrollar la flora intestinal que necesitan para digerir alimentos fibrosos. Lamentablemente, en los Estados Unidos, los reptiles, especialmente las iguanas, se han popularizado de tal manera que la importación anual de estos animales actualmente roza en el millón, y el aumento de casos de salmonelosis en niños pequeños ha sufrido un aumento paralelo al de la importación. Los Centros para el Control y la Prevención de Enfermedades (CDC), con sede en Atlanta, ha publicado recomendaciones muy estrictas sobre la manipulación de reptiles domésticos: las personas que tienen un riesgo aumentado de infección debido, por ejemplo, a un estado inmunodeprimido no deben tener contacto con reptiles. Además, se prohíbe tener estos animales en guarderías infantiles o en sitios donde se preparan alimentos (26).

La erliquiosis canina, que es común en zonas tropicales y subtropicales, también se encuentra en algunas partes de Europa y América del Norte. En la parte meridional de Europa, la infección la transmite principalmente el vector Rhipicephalus sanguineus. El agente causal detectado en los casos caninos de más reciente notificación ha sido E. chaffeensis (27). La infección por E. ewingii suele ser menos grave. En los cuatro casos humanos causados por $E$. ewingii, todos los pacientes se habían expuesto a la picadura de garrapatas y habían tenido contacto con un perro poco antes de la aparición de los síntomas. Uno de los perros tenía una infección asintomática, lo cual apunta hacia la posibilidad de que el perro sea un reservorio de la infección humana (18).

\section{CONCLUSIONES}

Es un hecho prácticamente seguro que nuevas zoonosis serán descubiertas en el futuro próximo, especialmente mediante el uso de las nuevas técnicas de biología molecular, tales como la RCP y la secuenciación del ADN. Los animales salvajes siguen siendo un gran reservorio de agentes patógenos que aún no se han identificado. No obstante, casi todas las zoonosis que afectan al ser humano, con la excepción de las que son transmitidas por vectores, suelen ser transmitidas por animales domésticos, especialmente cuando se trata de especies exóticas. En la mayor parte de los casos, las zoonosis bacterianas emergentes podrían prevenirse fácilmente mediante medidas básicas de higiene y la aplicación del sentido común. No cabe duda de que la desorganización y la transición a manos privadas de muchas agencias de salud pública en varias partes del mundo a fines del siglo pasado han contribuido a la aparición y reaparición de muchas zoonosis bacterianas.

\section{SYNOPSIS}

\section{Emerging bacterial zoonoses}

Different zoonoses whose etiologic agents were previously unknown have been identified in recent years as a result of new diagnostic techniques in molecular biology and improved epidemiological surveillance systems. Several factors have facilitated the emergence of new diseases and the reemergence of already known diseases, which were thought to be under control. They include the following: (1) environmental changes, such as deforestation and droughts, which allow etiologic agents and their vectors to thrive, (2) industrial food production for widespread consumption, which allows disease agents to spread quickly, (3) certain risky human habits, especially among immuno-compromised persons, such as having exotic pets at home, and (4) the adaptation of etiologic agents to new environmental conditions through the development of drug resistance. Other factors, such as the disruption of established health systems during the process of privatization, have also contributed to the situation. In most cases, bacterial zoonoses can be prevented through good personal and domestic hygiene and common sense.

This report is not an exhaustive presentation of all emerging and reemerging bacterial zoonoses. Rather, it aims to illustrate, through numerous examples, the various factors that have contributed to the epidemiological changes that have taken place since the latter part of the twentieth century.

\section{REFERENCIAS}

1. Meslin FX. Surveillance and control of emerging zoonoses. World Health Stat Q 1992;45:200-207.
2. Meslin FX. Global aspects of emerging and potential zoonoses: a WHO perspective. Emerg Infect Dis 1997;3:223-228.
3. Morse SS. Factors in the emergence of infectious diseases. Emerg Infect Dis $1995 ; 1: 7-15$ 
4. Woodford MH, Rossiter PB. Disease risks associated with wildlife translocation projects. Rev Sci Tech Off Int Epiz 1993:12:115-135.

5. Daszak P, Cunningham AA, Hyatt AD. Emerging infectious diseases of wildlife: threats to biodiversity and human health. Science 2000;287:443-449.

6. Glaser CA, Angulo FJ, Rooney JA. Animal-associated opportunistic infections among persons infected with the human immunodeficiency virus. Clin Infect Dis 1994;18:14-24.

7. MacArthur RD, Lopez CE. Bordetella bronchiseptica infection in human immunodeficiency virus-infected patients. Clin Infect Dis 1999;28:1095-1099.

8. Ford DB. Bordetella bronchiseptica has zoonotic potential. Topics Vet Med 1995; 6:18-22.

9. Woodard DR, Cone LA, Fovedt K. Bordetella bronchiseptica in HIV-infected persons. [Carta]. Clin Infect Dis 1994;20: 193-194.

10. Angulo FJ, Glaser CA, Juranek DD, Lappin MR, Regnery RL. Caring for pets of immunocompromised persons. J Am Vet Med Assoc 1994;205:1711-1718.

11. Lion C, Escande F, Burdin JC. Capnocytophaga canimorsus infections in humans: review of the literature and case report. Eur J Epidemiol 1996;12:521-533.

12. Underman AE. Bite wounds inflicted by dogs and cats. Vet Clin North Am 1987; 17:195-207.
13. Krol-Van Straaten MJ, Landheer JE, De Maat CEM. Capnocytophaga canimorsus (formerly DF-2) infections: review of the literature. Nether J Med 1990;36: 304-309.

14. Chomel BB, Boulouis HJ, Gurfield AN, Helller R, Piemont Y, Pilet C. Maladie des griffes du chat et infections associeés. Bull Acad Natle Med 1997;181: 441-450.

15. Sigal LH, Curran AS. Lyme disease: a multifocal worldwide epidemic. Ann Rev Pub Health 1991;12:85-109.

16. McQuiston JH, Paddock CD, Holman $\mathrm{RC}$, Childs JE. The human ehrlichioses in the United States. Emerg Infect Dis 1999;5:635-642.

17. Perez M, Rikihisha Y, Wen B. Ehrlichia canis-like agent isolated from a man in Venezuela: antigenic and genetic characterization. J Clin Microbiol 1996;34: 2133-2139.

18. Buller RS, Arens M, Hmiel SP, Paddock CD, Summer JW, Rikhisa Y, et al. Ehrlichia ewingii, a newly-recognized agent of human ehrlichiosis. N Engl J Med 1999;341:148-155.

19. Chen SM, Dumler JS, Bakken JS, Walker DH. Identification of a granulocytic Ehrlichia species as the etiologic agent of human disease. J Clin Microbiol 1994;32: 589-595.

20. Walker DH, Dumler JS. Emergence of the ehrlichioses as human health problems. Emerg Infect Dis 1996;2:18-29.

21. Raoult D, Tissot-Dupont H, Chicheportiche C, Peter O, Gilot B, Drancourt M.
Mediterranean spotted fever in Marseille, France: correlation between prevalence of hospitalized patients, seroepidemiology, and prevalence of infected ticks in three different areas. Am J Trop Med Hyg 1993;48:249-256.

22. Font-Creus B, Bella-Cueto F, EspejoArenas E, Vidal-Sanahuja R, MunozEspin T, Nolla-Salas M, et al. Mediterranean spotted fever: a cooperative study of 227 cases. Rev Infect Dis 1985;7: 635-642.

23. Higgins JA, Radulovic S, Schriefer ME, Azad AF. Rickettsia felis: a new species of pathogenic rickettsia isolated from cat felas. J Clin Microbiol 1996;34:671-674.

24. Perry RD, Fetherston JD. Yersinia pestis: etiologic agent of plague. Clin Microbiol Rev 1997;10:35-66.

25. Woodward DL, Khakhria R, Johnson WM. Human salmonellosis associated with exotic pets. J Clin Microbiol 1997; 35:2786-2790.

26. Centers for Disease Control and Prevention. Reptile-associated salmonellosisselected states, 1994-1995. MMWR 1995; 44:347-350.

27. Breitschwerdt EB, Hegarty BC, Hancock SI. Sequential evaluation of dogs naturally infected with Ehrlichia canis, Ehrlichia chaffeensis, Ehrlichia equi, Ehrlichia ewingii, or Bartonella vinsonii. J Clin Microbiol 1998;36:2645-2651.

\section{Zoonoses and Communicable Diseases Common to Man and Animals, 3. ${ }^{\text {rd }}$ edition. Vol. I. Bacterioses and Mycoses}

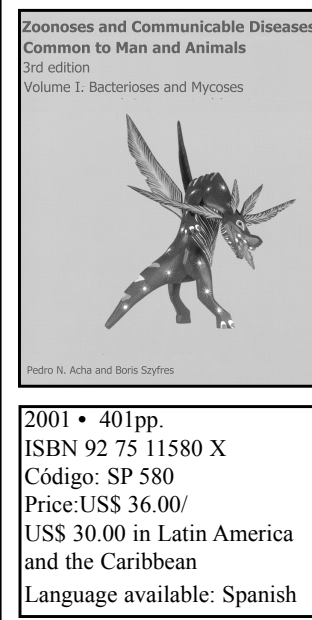

This popular book, useful for schools of public health, medicine, and veterinary medicine, as well as public health and animal health institutions, offers the latest information on zoonoses and zoonotic diseases in Latin America and the rest of the world. Thanks to new technologies and advances in epidemiology, ecology, and other biological and social sciences, great progress has been made regarding these diseases since the first edition was published in 1977. This edition is the first to be published as three volumes: the first includes bacterioses and mycoses; the second, chlamydioses, rickettsioses, and viroses; and the third, parasitic zoonoses.

This essential work also includes maps, tables, and figures that help explain the transmission cycle and the geographic distribution and prevalence of many of these diseases.

\begin{tabular}{|c|}
\hline $\begin{array}{c}\text { Volume II (Chlamydioses, Rickettsioses, and Viroses) and Volume III (Parasitic } \\
\text { Zoonoses) will be available soon }\end{array}$ \\
$\qquad \begin{array}{c}\text { http://publications.paho.org } \bullet \text { Fax: (301) 206-9789 • E-mail: paho@pmds.com } \\
\text { PAHO/WHO Office in your country }\end{array}$ \\
\hline
\end{tabular}

